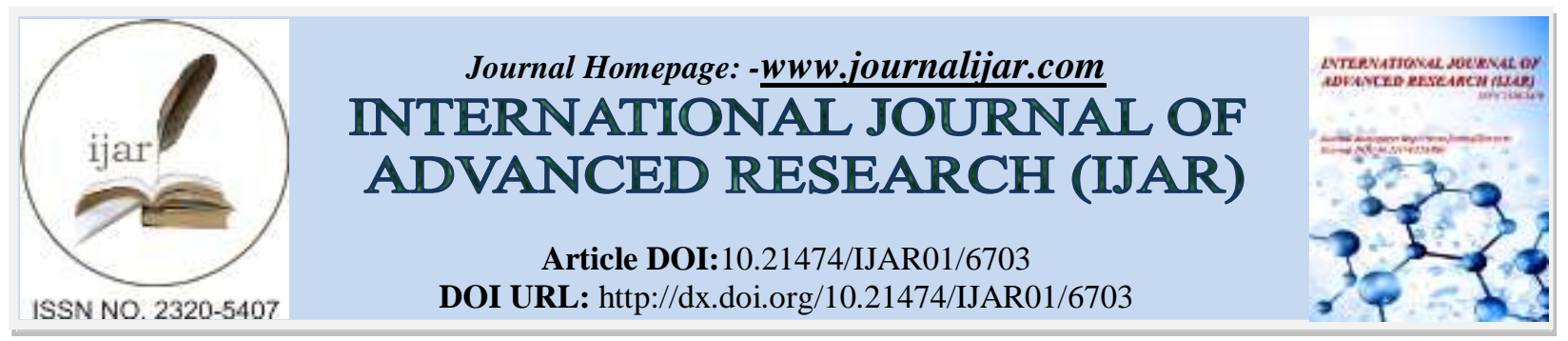

RESEARCH ARTICLE

\title{
SYNDROME DE POLAND: À PROPOS DE DEUX OBSERVATIONS.
}

H. Doulhousne, M. El ghaidi, M. Benzalim and H. Jalal.

Service de radiologie, Hôpital mère-enfant, CHU Mohammed VI, Marrakech, Maroc.

\section{Manuscript Info}

(.........................

Manuscript History

Received: 09 January 2018

Final Accepted: 11 February 2018

Published: March 2018

Keywords:-

Poland syndrome - imaging - diagnosis.

\section{Abstract}

The syndrome of Poland is a rare congenital malformation, grouping, to varying degrees, musculoskeletal abnormalities of the chest wall and abnormalities of the ipsilateral upper limb. We report two observations collected of this rare syndrome whose etiology remains uncertain. It seems that a defect of vascularization during the fetal development is at the origin of the described anomalies. CT is the exam of choice, allowing a complete assessment of the lesion, to look for the associated malformations and to guide the therapeutic attitude which varies according to the sex and the severity of the attack.

Copy Right, IJAR, 2018,. All rights reserved.

\section{Introduction:}

Le syndrome de Poland est une malformation congénitale rare qui se caractérise par des anomalies de la paroi thoracique associée ou pas à des anomalies du membre supérieur homolatéral. L'imagerie, notamment le scanner, permet de décrire ces anomalies, de chercher d'autres malformations associées et de guider la prise en charge thérapeutique.

\section{Observations:}

\section{Observation 1:}

Fillette de 7 ans, suivie pour dépression de l'hémithorax gauche (Figure 1). Une TDM thoracique est réalisée, objectivant une agénésie des faisceaux sterno-costaux du muscle grand pectoral gauche avec agénésie totale du petit pectoral. Ceci, sans anomalies osseuses des côtes homolatérales (Figure 2).

\section{Observation 2:}

Garçon de 13 ans, suivi pour déformation de l'hémithorax droit, chez qui la TDM thoracique a mis en évidence une agénésie totale des deux muscles pectoraux droits avec hypoplasie de l'arc antérieur de la $3{ }^{\text {ème }}$ cote homolatérale (Figures 3 et 4).

Les anomalies décrites ci-dessus faisaient évoquer le syndrome de Poland. De ce fait, un bilan malformatif fait d'échographie abdominale et de radiographies des mains est réalisé chez nos deux patients, n'objectivant aucune malformation associée, notamment rénale ou des os des membres supérieurs. 


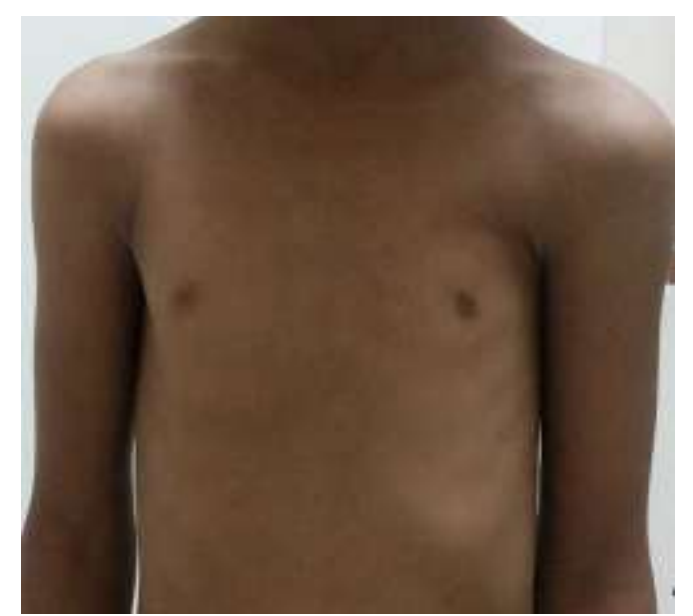

Figure 1 :- Aspect Clinique de la dépression de l'hémichamp thoracique gauche.

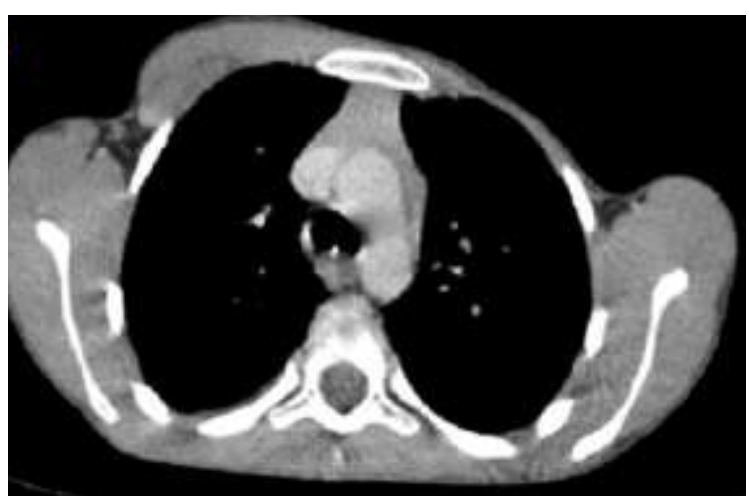

Figure 2: Agénésie des muscles pectoraux gauches sans anomalies costales associées en coupe axiale TDM.

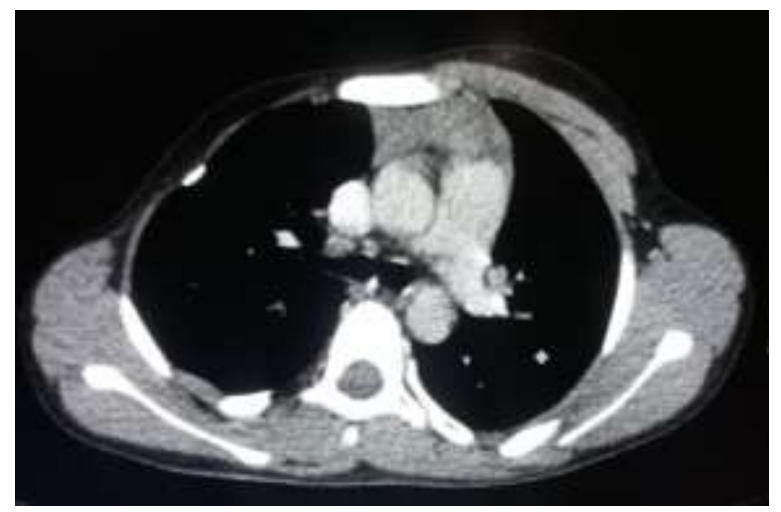

Figure 3: Agénésie totale des muscles pectoraux droits avec hypoplasie de l'arc antérieur de $3^{\text {ème }}$ cote en regard en coupe axiale TDM. 


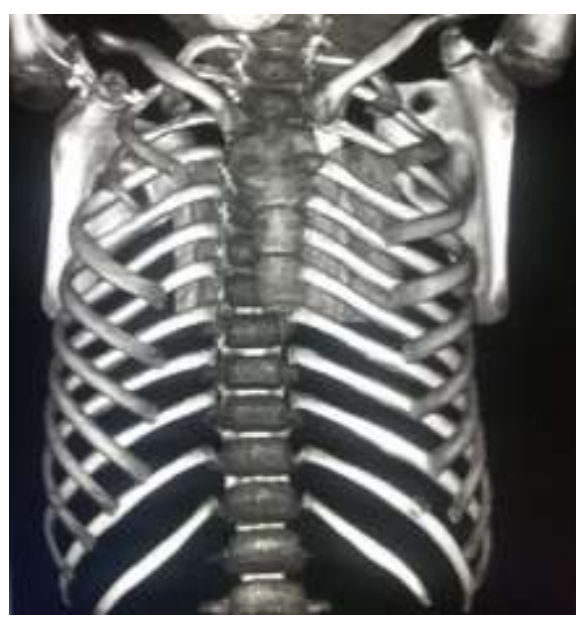

Figure 4: Reconstruction coronale 3D $(\mathrm{TDM})$ de la cage thoracique avec hypoplasie de l'arc antérieur de la $3^{\text {ème }}$ cote droite.

\section{Discussion:-}

Le syndrome de Poland est une malformation congénitale rare, regroupant des anomalies musculo-squelettiques de la paroi thoracique et du membre supérieur homolatéral [1-2]. Il a été décrit pour la première fois par Alfred Poland en 1841 [3]. Il survient habituellement de façon sporadique, seuls quelques cas familiaux ont été rapportés [1,3]. Son incidence est estimée de 1 à 3 par 100.000 nouveau-nés avec une nette prédominance masculine [1,4]. Le côté droit étant le plus affecté dans $75 \%$ des cas rapportés [1]. Son étiopathogénie demeure inconnue. Plusieurs théories ont été avancées. Mais, il semble que l'interruption du flux sanguin fœtal au cours de la sixième semaine de gestation pourrait expliquer l'agénésie du muscle pectoralis major et les autres variantes du syndrome [3,5].

Dans sa forme complète, ce syndrome associe une agénésie du pectoralis major à une symbrachydactylie de la main homolatérale (malformation associant, à des degrés divers, hypoplasie des doigts et syndactylie). Plusieurs variantes lui ont été décrites incluant l'agénésie totale des deux muscles pectoraux ou isolée du muscle petit pectoral, agénésie de la glande mammaire, hypoplasie des côtes, hypoplasie de l'omoplate et de ses muscles, voire une dextrocardie lorsque l'atteinte est à gauche, une agénésie rénale, du diaphragme ou encore des malformations intestinales [6]. L'atteinte du membre supérieur n'est rencontrée que dans 12\% des syndromes de Poland décrits [7]. L'aspect d'une petite main palmée avec absence de phalange intermédiaire des doigts centraux (deuxième et troisième) est caractéristique.

La TDM est l'examen de choix pour le diagnostic de ce syndrome. Elle permet d'évaluer l'hypoplasie musculaire, de révéler toutes les anomalies décrites au cours de ce syndrome, mais aussi les anomalies osseuses associées de la cage thoracique de façon plus précise que la radiographie thoracique [3,4]. Elle permet aussi de guider l'attitude thérapeutique. La réalisation de l'IRM dans le but d'une meilleure analyse de l'atteinte musculaire est discutée [4]. L'échographie mammaire doit être réalisée chez les filles en période pubertaire à la recherche d'une agénésie associée de la glande mammaire.

Le traitement consiste à réaliser une reconstruction chirurgicale par transposition du muscle grand dorsal intacte pour combler le vide dû à l'absence du muscle grand pectoral chez des patients de sexe masculin vers l'âge de 13-14 ans [3]. Chez les femmes, le développement anormal de la glande mammaire est à l'origine de préjudices esthétique et psychologique, un traitement précoce par prothèse est alors envisagé une fois le développement des seins est achevé [3].

\section{Conclusion:-}

Le syndrome de Poland est une malformation congénitale rare, associant à des degrés divers, des malformations thoraciques et des malformations du membre supérieur homolatéral. Le scanner permet de confirmer le diagnostic, de dresser le bilan lésionnel complet et de guider la stratégie thérapeutique appropriée, tenant compte du sexe, de l'âge et de la sévérité de l'atteinte. 


\section{Conflit d'intérêt:}

Les auteurs déclarent ne pas avoir de conflit avec ce manuscrit.

\section{Bibliographie:-}

1. Frioui S, Khachnaoui F. Poland's syndrome. Pan African Medical Journal. 2015; 21 (1): 294.

2. Iyer R, Parisi M. Multimodality imaging of poland syndrome with dextrocardia and limb anomalies. Clinical Nuclear Medicine. 2012;37(8):815-816.

3. Kapetanakis S, Papadopoulos C, Triantafilidis A, Fiska A, Agrogiannis N, Maria D, Panagiotou P. Muscle abnormalities of the chest in Poland's syndrome: variations and proposal for a classification. Surg Radiol Anat. 2012;34(1):57-63.

4. Chichery A, Jalbert F, Foucras L, Grolleau J-L, Chavoin J-P. Syndrome de Poland. EMC, Techniques chirurgicales-Chirurgie plastique reconstructrice et esthétique. 2006; 45-667-E:1-17.

5. Legbo JN. Poland's syndrome: report of a variant. J Natl Med Assoc. 2006;98(1):97-9.

6. Chowdhury K, Chakrabortty R, Gope S. Poland's syndrome: a case report and review of literature. J Pak Med Assoc. 2015;65(1):87-9.

7. Foucras L, Grolleau J.L, ChavoinJ.P.Syndrome de Poland et malformations de la main : à propos d'une série clinique. Annales de Chirurgie Plastique Esthétique. 2005 ; 50(2) :138-14 